\title{
EFFECTS OF TERMINAL SIZE, YARD BLOCK ASSIGNMENT, AND DISPATCHING METHODS ON CONTAINER TERMINAL PERFORMANCE
}

\author{
Anne Kathrina Schwientek \\ Ann-Kathrin Lange \\ Carlos Jahn \\ Institute of Maritime Logistics \\ Hamburg University of Technology \\ Am Schwarzenberg-Campus 4 \\ Hamburg, 21073, GERMANY
}

\begin{abstract}
Given the growth in ship size and increasing demands, it is essential for seaport container terminals to make well-founded tactical and operational decisions. Here, horizontal transport is an important element of container terminals, connecting seaside handling with container storage. An efficient design of horizontal transport, especially the assignment of vehicles to orders, strongly influences the terminal performance. Most existing scientific studies vary only individual parameters or dispatching strategies neglecting different terminal targets. This study approaches this research gap. In a discrete-event simulation model, the influence of various terminal parameters on dispatching strategies is examined, taking into account the terminal targets. The two most influencing terminal parameters, terminal size and yard block assignment to containers, are analyzed in detail. The results show that the best choice of yard block assignment and dispatching method for a given terminal size depends on the combination of both parameters and the aspired targets.
\end{abstract}

\section{INTRODUCTION}

Driven by constantly increasing handling volumes and ever larger ships, seaport container terminals face the challenge to handle containers as quickly and efficiently as possible. As nodes in maritime logistic networks, they are mainly used to transport standardized containers between sea-going vessels and means of hinterland transport or between different sea-going vessels. The most common sizes of containers are 20'- and 40'-containers, measured in Twenty-foot Equivalent Unit (TEU), which are used worldwide as a uniform measure for the capacity of a container. The handling processes can be divided into import, export and transshipment processes (Vis and de Koster 2003; Gharehgozli et al. 2016) as displayed in Figure 1. At the quay, containers are usually unloaded from a ship or loaded onto a ship by ship-to-shore cranes (STS). Transport between the quay side and the terminal's own storage area ("horizontal transport") is carried out with different equipment such as tractor-trailer units (TT) or straddle carriers (SC). Horizontal transport, thus, represents the interface between the sea side of the terminal and the land side. In the storage area ("yard") - depending on the equipment type for horizontal transport - the equipment is either loaded/unloaded by gantry cranes or the container is placed or picked up. Gantry cranes can be rubber-tyred gantry cranes (RTGs) or rail-mounted gantry cranes (RMGs).

The most common container handling system is the RTG/TT system (Wiese et al. 2009). In the RTG/TT system, STS unload containers from a ship and set them down on a vehicle (i.e., a TT) that is standing by. The TT then transports the container to the yard, where an RTG lifts the container from the vehicle into the storage block (Brinkmann 2011). The loading of a ship is carried out in the reverse direction. External truck handling is also carried out by RTGs, sometimes supplemented by a few reach stackers (RS) for reasons of flexibility. Rail transshipment is carried out by RS when the volume of rail traffic is rather 
Schwientek, Lange, and Jahn

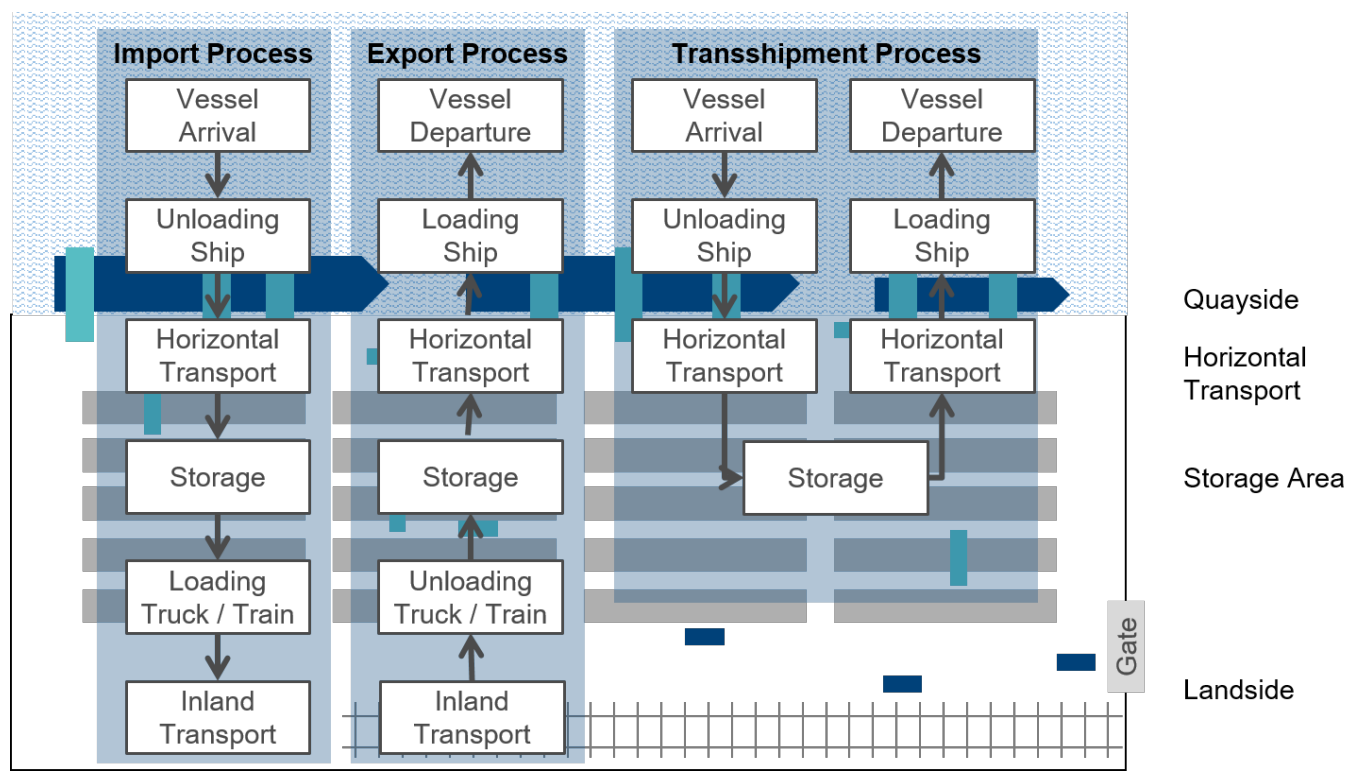

Figure 1: Processes on a container terminal.

low. RMGs alongside the rail tracks are used when the volume of rail traffic is higher. Among others, Sines Container Terminal in Portugal, MSC Terminal Valencia in Spain, and Container Terminal Klaipeda in Lithuania apply the RTG/TT system.

The RTG/TT system has a high stacking density of approximately $1000 \mathrm{TEU} / \mathrm{ha}$ and is, therefore, suitable for high handling rates in a rather small space (Brinkmann 2011). At the same time, the system is flexible as the RTGs can be moved between the storage blocks. A disadvantage of this system is that TT are passive vehicles needing to be unloaded and loaded, so that container transfer is potentially delayed. Furthermore, terminal operations can be disrupted by external trucks that drive into the storage area for container transfer.

There are different approaches to organize container terminal operations in a way that allows for handling the ships efficiently. One of them is a fast, reliable, and efficient horizontal transport. In order to achieve these objectives, there are some important decisions to be taken. In addition to decisions on which equipment is used and how many vehicles are reasonable for smooth terminal operations, the main question that arises is how to best assign vehicles and container transport orders (Carlo et al. 2014). The assignment aims at the most efficient combination of container transport orders and vehicles for a defined time horizon. Thereby, various terminal objectives should be taken into account. These include, among others, the continuous operation of the STS cranes or the minimization of the distances traveled in horizontal transport.

For the assignment of orders for container transports and vehicles, there are - from a scientific perspective - two essential approaches: Dispatching and scheduling (Bian et al. 2015). The dispatching approach is dynamic and based on priority rules that specify how the next job is selected. The assignment is triggered event-driven, e.g., when a vehicle becomes available, and very flexible. Therefore, it is possible to react to short-time changes and stochastic fluctuations, because ad hoc in the respective situation, it is decided which transport order is to be executed. Thus, this approach does not seek an optimum by comparing several possible solutions, but always selects one solution based on priority rules.

In contrast, the scheduling approach is static in nature. It generates a longer-term plan for order assignment, based on estimated arrival and handling times and planned start and destination locations for each transport order. This plan is usually optimized by using mathematical methods and, thus, seems to be superior to the rule-based dispatching approach. However, the quality of the optimized plan depends on the quality of the time estimates. Due to the stochastic nature of the terminal processes, a large number 
of influencing factors change the conditions of the assignment decisions within minutes (Grunow et al. 2006). This can lead to an optimized longer-term plan that is no longer optimal. This study, therefore, focuses on the dynamic dispatching approach.

In practical terminal operation, there is another approach: the fixed assignment. In this case, a group of vehicles and personnel is assigned to one STS and shall only execute transport orders that belong to that STS. As pragmatic as this approach is, it also appears inefficient, since the fixed assignment means that a large proportion of journeys are empty trips and neither distances nor handling times are minimized.

In the following, a short literature review on dispatching in horizontal transport is presented to derive the research question (Section 2). In Section 3, the research approach, which combines a preliminary investigation and a detailed analysis, is discussed in detail. Section 4 presents the results of the detailed analysis.

\section{LITERATURE REVIEW}

The challenges to the design, efficiency, and productivity of container terminals are the subject of farreaching scientific studies, which are analyzed in different publications such as Vis and de Koster (2003), Carlo et al. (2014), and Gharehgozli et al. (2016). While Carlo et al. (2014) refer more generally to transport operations at container terminals, Stahlbock and Voß (2008) consider in particular routing problems of vehicles at container terminals. The specific topic of dispatching in horizontal transport of container terminals is investigated in many scientific publications (Schwientek et al. 2017). A number of publications analyze the effects of different dispatching methods at container terminals. Dynamic dispatching methods such as "next vehicle" or "lowest capacity utilization" are examined by e.g. Grunow et al. (2006) and Tao and Qiu (2015). Corman et al. (2016) and He et al. (2015), for example, follow the static approach to formulate a mathematical model and solve it either exactly or with heuristics such as genetic algorithms or tabu search.

The respective publications usually develop their own assignment method or compare methods for a specific terminal. Several authors vary within the sensitivity analysis some terminal parameters and investigate the effect on the achieved results (Grunow et al. 2006; Klerides and Hadjiconstantinou 2011; Zeng et al. 2009). Zeng et al. (2009) and Liu and Ioannou (2002) show that the number of vehicles influences the success of the dispatching methods. Thus, there are terminal parameters, such as the number of vehicles, which influence the choice of an dispatching method. The decision for a dispatching method should take into account that another method may be more effective in achieving the terminal objectives.

This raises the question of which terminal parameters actually influence the success of a dispatching method. Furthermore, Dulebenets (2016) states that dispatching methods can have varying degrees of success according to the evaluation criterion. This means that also the definition of terminal objectives influences the success of different dispatching methods. The connections between terminal objectives, terminal parameters, and dispatching methods have not yet been systematically investigated.

This leads to the research question, which connections exist between dispatching methods for orders in horizontal transport, terminal parameters, and the performance of container terminals? Thereby, the new contribution of this study is that it analyses on a broad generic level which dispatching method is best suited to achieve different terminal objectives depending on the characteristics of different terminal parameters. In the following, it will, therefore, be investigated on a generic level which dispatching method should be used to best achieve defined terminal objectives under the influence of which terminal parameters.

\section{METHODOLOGY AND SOLUTION APPROACH}

The use of simulation studies in the development of ports and in particular of container terminals is becoming increasingly important (Dragovic et al. 2017). This development is based on two main reasons:

(1) A container terminal is a dynamic system. Stochastic influences are, for example, the arrival rates of the containers on both the sea and land side, the handling times of the equipment (in the case of a STS, 
for example, depending on the wind conditions, the skill of the crane operator, the position of the container on the ship and thus the visibility of the container) or the failure rates of the equipment.

(2) Although the order assignment in the horizontal transport of container terminals is also often examined with the aid of mathematical methods, only a few parameters are then varied and the complexity of the model and, thus, the computing time of the solution methods increases rapidly.

First, a base terminal implemented in the simulation model is described as a conceptual model. The base terminal serves as a comparison model for all parameter variations. In the preliminary study, a number of terminal parameters is varied ceteris paribus to identify the most relevant ones. Furthermore, five different dispatching methods are compared to identify particularly successful ones. Based on the preliminary study comprising 160 experiments with 50 replications each, the experiment plan for the detailed study is derived.

\subsection{Simulation Model}

The simulation model is based on a typical RTG/TT container terminal as illustrated in Figure 2. It is implemented in Tecnomatix Plant Simulation. The model shows the horizontal transport with the adjacent areas. On the upper side of the figure is the quay on the sea side with the STS and on the bottom side the storage area with the RTGs. The dashed red line shows the system boundaries of the simulation model.

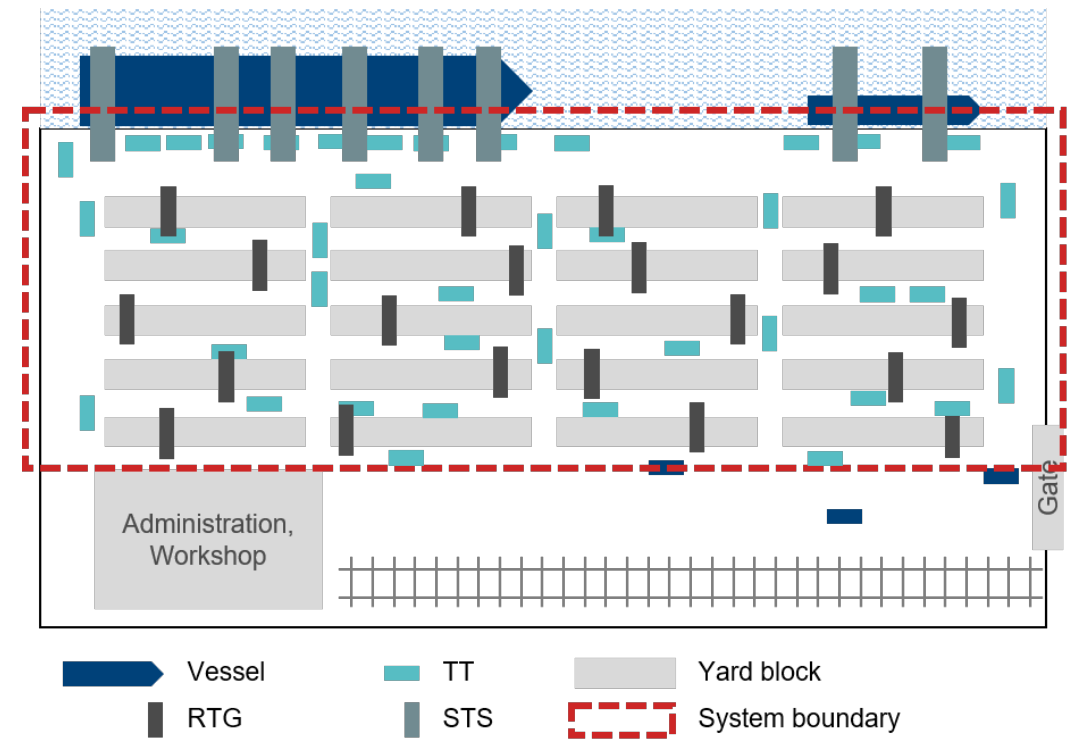

Figure 2: Layout of the 1.6 Mio.TEU reference terminal.

On both the sea and the land side, the model contains sources and sinks for the containers. The sources are controlled via a sailing list, i.e., whenever a ship docks at the terminal according to the sailing list, the respective sources generate the corresponding number of import and export containers. Both the optimization of the berths and the optimized STS deployment planning are outside the system boundaries. Thus, the STS are assigned to a ship already in the sailing list. After the containers have been handled and transported to the other side, they leave the simulation model. For the storage area, this implies that the stock of the yard blocks is not considered further. The land side interface, the gate, is not modeled, since for the base terminal it is assumed that truck arrivals will not interfere with the yard operation for ship handling. This assumption is justified by the fact that ship handling has a higher priority for the terminals than truck handling according to terminal operators. This is verified in separate simulation experiments.

The core process of the simulation model includes the unloading and loading of containers at the quay side. After a vessel has been created, the import containers are unloaded by several STS, then - when a STS has completed the unloading process - the export containers are loaded onto the vessel. When all 
the STS are finished, the ship sets off again and the corresponding section of the quay wall is free for the next ship. The assumption that first all import containers are discharged and then all export containers are loaded contains a certain simplification. In reality, an STS is responsible for several loading bays on the ship and this results in several load or unload sequences.

At the beginning of the simulation, a defined number of vehicles for horizontal transport is generated and distributed to different parking positions in the area of the quay side. In case of a fixed assignment between vehicle and STS, a so-called base STS is assigned to the vehicle and the vehicles are distributed evenly to the STS used. If there are new active orders or if an unassigned vehicle passes one of the sensors distributed along the routes, the order search is triggered in the model. After an order has been assigned, the vehicle drives to the start location of the order (to the STS in case of the unloading process), is loaded with the container by the STS there, takes over the destination of the order as a new destination and drives there. At the destination, the container is unloaded from the vehicle by an RTG and the order search is triggered again. If no active and, therefore, open transport order is found or when no order can be assigned due to restrictions, the vehicle drives to the nearest parking position. Parked vehicles resume operation during the simulation when a new order assumes the status active. Parking positions for TT are located between the quay wall and the yard blocks. There is a parking lot at every second STS with a restricted capacity. This means that the parking positions are evenly distributed between the start locations of the orders.

To parametrize the base terminal, the following assumptions have been made. They are based on expert interviews, anonymized company information and an in-depth literature search of other simulation studies. In the base model, the simulated terminal has a capacity of about 1,600,000 TEU/a with a TEU factor of 1.6, which is about the average value of the most common $80 \%$ of RTG terminals. The capacity of a terminal is the theoretical maximum number of containers that can be handled on the quay per year. It is limited by the length of the quay wall and the number and productivity of available STS. The terminal capacity is also influenced by the size of the storage area. The TEU factor reflects the ratio of 20 '- to 40 '-containers. A TEU factor of 1.6 implies $60 \% 40$ '-container and $40 \% 20$ '-container. The quay length is $800 \mathrm{~m}$, eight STS are installed. The handling time of all STS is triangularly distributed $(120 \mathrm{~s}, 80 \mathrm{~s}$, $180 \mathrm{~s}$ ) and corresponds to an average productivity of 30 moves/h. For each STS the next three transport orders are known with certainty and can be assigned to a TT.

The yard consists of 20 blocks (6 TEU wide x 28 TEU long), which are aligned in parallel to the quay wall. The arrangement of the yard blocks often depends on the selected equipment. In each yard block one RTG is available for the sea side, i.e., with full load at the quay side 2.5 RTGs can be used per STS. This assumption is a simplification of reality. At a real terminal, two RTGs can operate simultaneously in one yard block and RTGs are able to change blocks. Nevertheless, the decisions which RTG is used where and when it changes the yard block are too complex and depend on too many factors. Implementing these decisions would result in a much more detailed modeling of the yard blocks and the storage strategies, which would shift the focus in the present study strongly from horizontal transport to the storage area. The handling time of the RTG is triangularly distributed $(240 \mathrm{~s}, 150 \mathrm{~s}, 360 \mathrm{~s})$ and corresponds an average productivity of 15 moves/h.

There are 40 TT for horizontal transport, each of which transports one or two containers at a speed of $30 \mathrm{~km} / \mathrm{h}$. 5 TT per STS is roughly equivalent to the usual TT number of real terminals, as it is a good balance between vehicle utilization and reliability in order to provide a continuous flow of containers to and from the STS. The number was verified in the preliminary study.

Only standard containers are considered. Other container types such as reefer or dangerous goods are not considered, because their special storage requirements do not influence the question. It is assumed that 20'-containers moved by an STS using a twin-lift also have the same storage location and are transported together. The topic of twin-lift transports is investigated in more detail in Schwientek et al. (2018).

The yard block assignment of containers is randomly evenly distributed over the terminal. Three different types of vessels arrive at the terminal: large deep-sea vessels, small deep-sea vessels and feeder 
vessels. The capacity utilization at the quay side is $75 \%$ in the base model. A utilization of $100 \%-$ including maneuvering times - would imply that the terminal is used at the quay side to its maximum capacity and that there are no equipment failures or significant delays in operations. A quay utilization of $75 \%$ means that the quay wall is occupied by moored ships $75 \%$ of the time. Thus, this sets the load of the system. The number of equipment from the terminal is not affected by the capacity utilization.

\subsection{Investigated Dispatching Methods}

Based on the literature analysis in Section 2, the dispatching methods are selected which are to be compared with each other in the simulation. Due to the dynamics of the terminal processes, the focus is on priority rules. Four representative methods for the most frequently used methods are compared: a time-, a distance-, an inventory-based, and a hybrid dispatching method. In addition, the fixed assignment is implemented as a typical practical solution tested in the simulation.

Since fixed assignment is often used in practice, it is examined in the present investigation as a comparative method. In the implementation, an STS is assigned to each vehicle at the beginning of a simulation run. That STS is referred to as base STS. If a vehicle is available, it searches the order list for an active order that is connected to the vehicle's base STS. Orders are assigned to an STS if they are handled by this STS. The distance-based method is a priority rule, which in various studies has proven to be more effective than other rules (Dulebenets 2016). In this method, the transport order is selected for a vehicle for which the distance between the current vehicle position and the start location of the order is the shortest. The time-based method selects the transport order for an available vehicle that has been waiting the longest for an assignment. This method thus focuses more on the smooth operation of the STS. Time-based dispatching methods are positively evaluated by Zeng et al. (2009), among others. Inventory-based dispatching is a method based on the method of Briskorn et al. (2006). This method is confirmed as successful by inter alia Choe et al. (2012). Like the time-based method, the inventory-based method aims at an operation of the STS as uninterrupted as possible. For this purpose, the inventory of an STS is defined as the number of currently assigned transport orders. For an STS in load mode, this corresponds to the number of containers that are currently being transported to this STS or that are being transported very soon by an already assigned vehicle, since it is already on its way to the container. In unloading mode, the inventory level corresponds to the number of vehicles that head to the STS to take over a container. The hybrid method is a combination of the other three dispatching methods. For this purpose, a score is calculated, which is made up of three components for the shortest distance, the longest waiting time and the lowest inventory.

Figure 3 shows an overview of the simulation results of all experiments in the preliminary study including the $95 \%$ confidence intervals. Two main output variables are shown: the average productivity of the STS in moves/h and the average distance traveled per container handled in meters. The mean values were calculated for each dispatching method over all 32 experiments in the preliminary study.

The dispatching method that leads to the best average result (e.g., the highest productivity or the shortest distance traveled) for each output variable is colored black. The lighter the color of the column for the respective dispatching method, the less effective the dispatching method is. The STS productivity as a key performance indicator of a container terminal is lowest on average over all experiments with a fixed assignment, about $2 \%$ higher with the distance-based method and about $4 \%$ higher with the other dispatching methods, with the time-based method performing slightly better.

The distance traveled per container as the second output variable is lowest for the distance-based and hybrid methods. The other dispatching methods result in 7-8 \% longer distances traveled on average. Thereby, the confidence intervals partly overlap. For terminals, longer distances are directly reflected in a correspondingly higher fuel consumption and thus influence the efficiency and the costs of the terminal. The distance-based method and the fixed allocation are, thus, comparable to or worse than the hybrid method on average of all experiments for both output variables. The time-based and the inventory-based method are similar in their results, although the time-based method usually scores slightly better. 


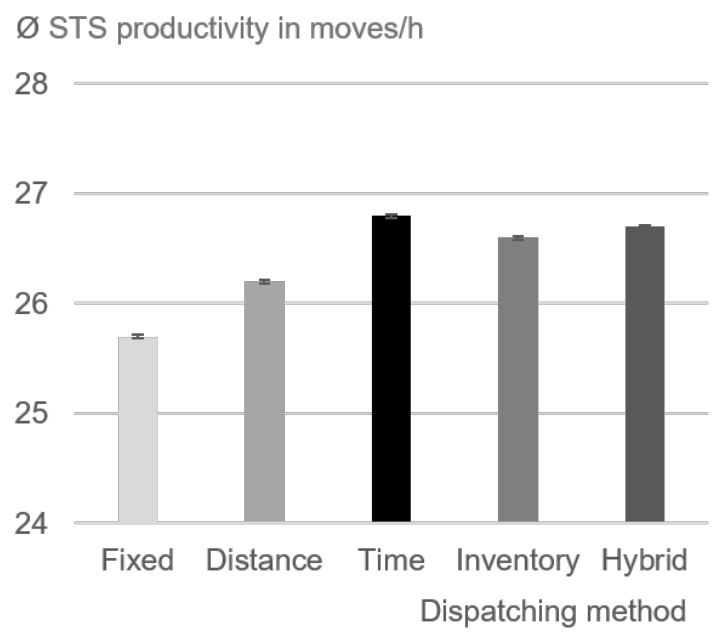

Best method
Second best method

Third best method $\varnothing$ Driven distance per container in $\mathrm{m}$

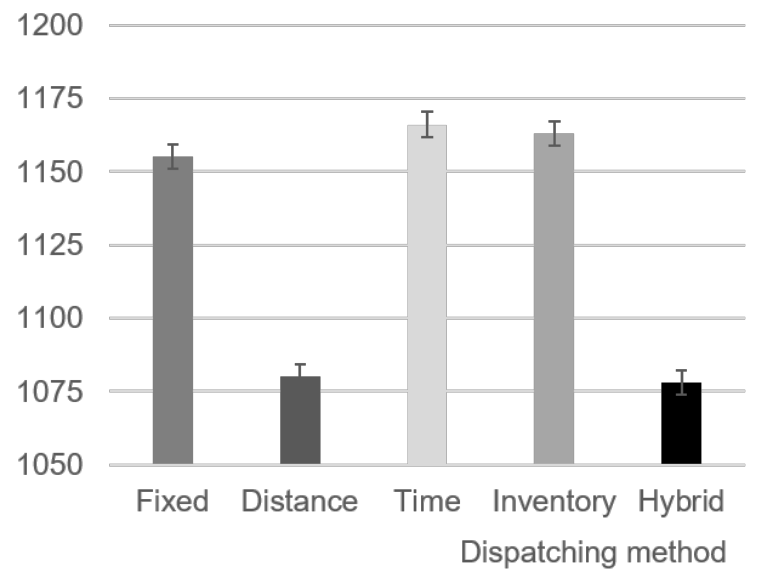

Fourth best method
Fifth best method

Figure 3: Comparison of different dispatching methods.

\subsection{Relevant Terminal Parameters}

The preliminary study also identifies the terminal parameters that influence the performance of the dispatching methods. Figure 4 summarizes the results of the preliminary study. Parts of this preliminary study can be found in Schwientek et al. (2018). For each terminal parameter investigated, the influence of the parameter on STS productivity and the distances traveled are shown. The influence on performance is quantified in each case with the percentage deviation from the best result in each case. This refers to the variance when only the dispatching method is changed in horizontal transport and all other parameters remain unchanged.

At first glance, the influence of terminal parameters such as the number of vehicles on the performance of the terminal appears comparatively high and, therefore, relevant. For the choice for the simulation study, however, it is above all relevant whether a change in the terminal parameter changes the ranking of the dispatching methods and, thus, a different dispatching method leads to higher performance.

The STS productivity shows to be influenced by many terminal parameters: These are the number of vehicles in horizontal transport, the yard block assignment to containers, the utilization of seaside capacity, the vessel sizes, the terminal size, the used equipment type in horizontal transport, the range of handling times and the handling rate of the STS. Apart from the number of vehicles, the influence is below $6 \%$ in all constellations examined. However, the ranking of the dispatching methods in relation to STS productivity is only marginally or not at all influenced by the terminal parameters investigated.

The distances traveled in horizontal transport are influenced by the number of vehicles, yard block assignment to containers, utilization of seaside capacity, vessel sizes, terminal size, used equipment type in horizontal transport, forecast horizon, and layout. Depending on the scenario, the influence can be up to $20 \%$. The yard block assignment to containers also influences the ranking order of the dispatching methods, that is, if the assignment is changed, a different dispatching method leads to shorter distances. It will, therefore, be examined more closely in the following simulation experiments. In addition, the terminal size will also be varied, since it influences both STS productivity and distances traveled, and it is precisely the combination of the terminal size and the yard block assignment that appears relevant.

\subsection{Experiment Plan}

Based on the selected dispatching methods (see Section 3.2) and terminal parameters (see Section 3.3), the experiment plan is created following a full factorial design. Thereby, the terminal size is determined 


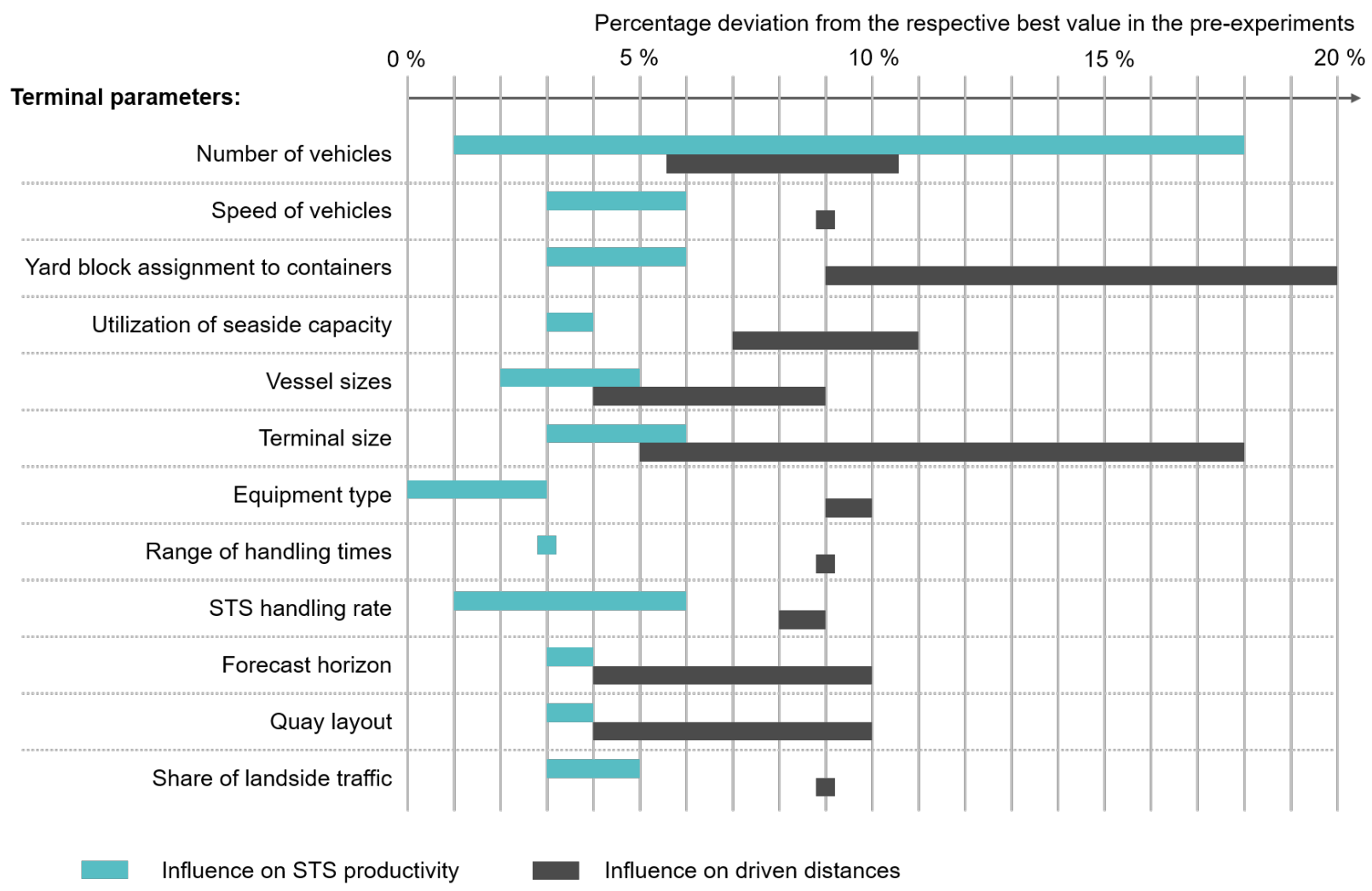

Figure 4: Results of the preliminary study.

by the maximum capacity of the quay. Four different terminal sizes are examined, which correspond to typical container terminals. The quay length of the base terminal is multiplied by $0.5,0.75$ and 2 , resulting in a quay capacity of $0.8,1.2,1.6$, and 3.2 million TEU/a. The number of STS, TT, and yard blocks is adjusted accordingly. The depth of the terminal is not changed.

For the yard block assignment, the container assignment is simplified to a storage block and not to a specific storage position in the block. In the experiment plan, four block assignment strategies are varied based on discussions with terminal experts: random, three, close, and mixed. For the random assignment, containers are randomly assigned equally distributed to all storage blocks. This assignment is primarily for the purpose of comparability. In practical operation, a few blocks are assigned to each ship. The following variants are based on this. For the three block assignment, three fixed storage blocks are assigned to each STS. They are defined before the simulation runs and stay the same. For the close block assignment, three fixed storage blocks are also defined for each STS, but they are located near the respective STS to minimize the travel distances. The mixed block assignment is a combination of three and close. There, an assignment to three nearby yard blocks is chosen for export containers and an assignment to three yard blocks nearby for import containers.

\section{RESULTS}

\subsection{Effects on the STS Productivity}

The average STS productivity is displayed in Figure 5. It increases with terminal size from 0.8 million TEU/a to 1.6 million TEU/a from an average of 25.9 moves/h to 26.7 moves/h. At the 3.2 million TEU/a terminal, the STS productivity remains at 26.7 moves/h on average for both dispatching methods. With a larger terminal, more vehicles are available providing more flexibility to avoid delays in STS operation. 


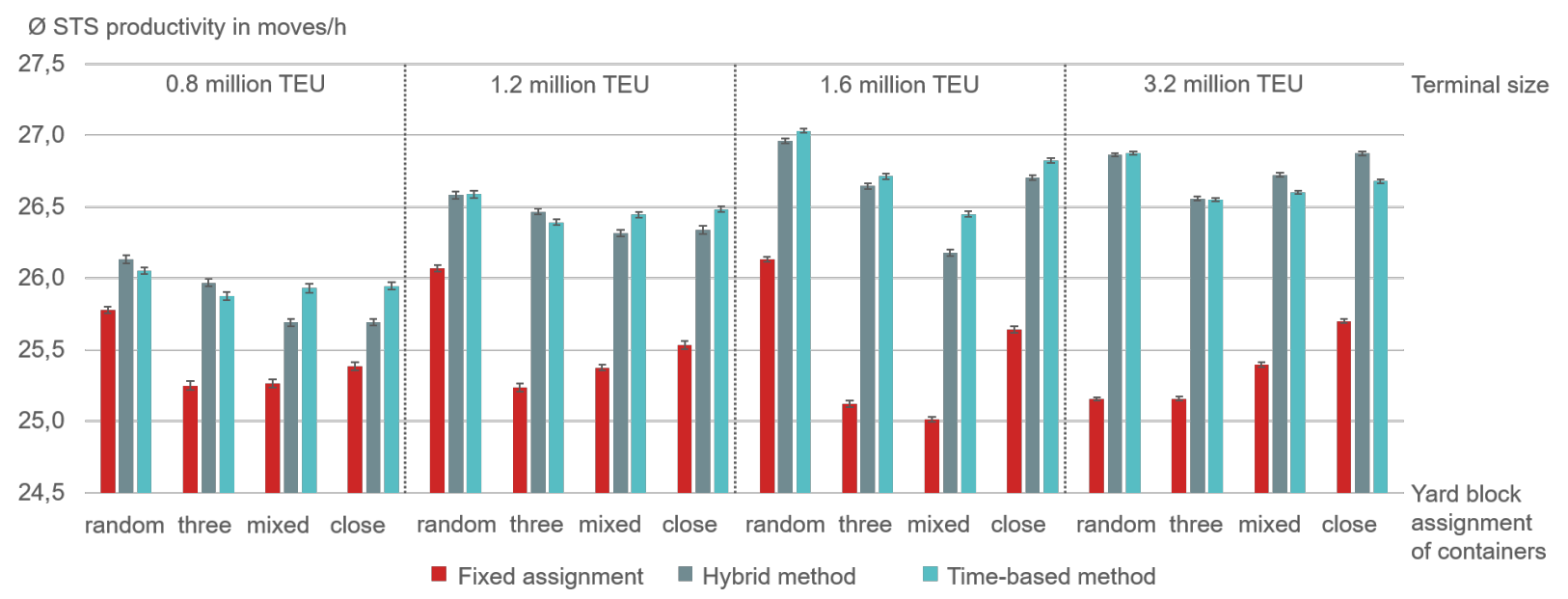

Figure 5: STS productivity depending on terminal size and yard block assignment.

The STS productivity is always 0.2 to 0.4 moves/h higher with the random yard block assignment than with the other yard block assignment strategies. This is most likely due to the fact that the random yard block assignment causes less delays in the yard area, because more yard blocks and thus more RTGs are in use. The assumption that there is an RTG in each yard block results in less waiting time in the yard area, which then also leads to waiting times at the STS. Thus, the higher STS productivity in random yard block assignment is offset by higher personnel costs, as all RTGs must be manned with a driver as soon as a ship is discharged or loaded and only one STS is in operation.

With fixed assignment, the mean value of STS productivity is constant in the range of 25.4 to $25.5 \mathrm{moves} / \mathrm{h}$. The increase in STS productivity, which can be observed with the time-based and hybrid method, is not so clear here. This results in the effect that the distance between the fixed assignment on the one hand and the time-based and hybrid method on the other hand increases with increasing terminal size. This means that the fixed assignment method leads to only minor productivity losses of less than $1 \mathrm{move} / \mathrm{h}$ for smaller terminals. One way to substantiate this observation is the fact that with the fixed assignment, five TT are always used for each STS. With the other dispatching methods, up to eight TT can work simultaneously for one STS, thus reducing waiting times as the TT belong to a large vehicle pool that executes all orders. Preliminary tests showed that it is necessary to limit the number of vehicles per STS to eight, otherwise congestion will occur, e.g., if only a few STS are active serving a small ship.

Even with the fixed assignment, random yard block assignment results in an average 0.5 to 0.8 moves/h higher STS productivity than the other yard block assignments within one terminal size. This is no longer the case for the largest terminal. There, with a fixed assignment in horizontal transport, the close yard block assignment has the highest productivity. This is probably caused by a sharp increase in the distances traveled, so that the transports take longer and the impact of a close yard block assignment is more significant.

In the case of the smallest terminal, the values for STS productivity are closest to each other. The range for the terminal is 0.9 moves/h and doubles to 2 moves/h for the 1.6 million TEU/a terminal. This corresponds to $3 \%$ and $8 \%$ of the average productivity for the respective terminal size. Thus, the relevance of the right choice of dispatching method and yard block assignment increases with the terminal size. This means for a larger deep-sea vessel, which, e. g., brings and collects 3,750 containers each and, thus, causes 7,500 handling moves being served by six STS, that each STS has to conduct 1,250 moves. With a productivity of 25 moves $/ \mathrm{h}$, a handling time of $50 \mathrm{~h}$, with 27 moves/h of only $46.3 \mathrm{~h}$ is achieved. The time saving of $3.7 \mathrm{~h}$ can be used to make up for ship delays or to slow down the ship and thus save fuel.

The time-based and the hybrid method lead to similar results in STS productivity for all yard block assignment strategies and terminal sizes and differ by a maximum of 0.3 moves $/ \mathrm{h}$. Neither yard block assignment strategy outperforms the other. The fixed assignment method for horizontal transport is on 
average 1 move/h and a maximum of 1.7 moves/h worse than the other two methods and is therefore dominated by the other two dispatching methods regardless of the yard block assignment.

\subsection{Effects on the Driven Distances}

In this study, the range of driven distances increases compared to the preliminary study (Figure 6). This is caused by the fact that the added experiments, which vary in yard block assignment, consistently lead to shorter distances and, thus, increase the range of results. The range of distances for TT increases to $120 \mathrm{~m}$ or $13 \%$ for the 0.8 million TEU/a terminal and to $987 \mathrm{~m}$ or $81 \%$ for the $3.2 \mathrm{million}$ TEU/a terminal. These results show that the distances traveled do not necessarily increase with terminal size. Rather, with the right choice of a dispatching method (hybrid or fixed assignment if necessary) and yard block assignment (close - as far as possible - or mix) it is possible to keep the distances traveled at a level comparable to a small terminal, even with a large terminal.

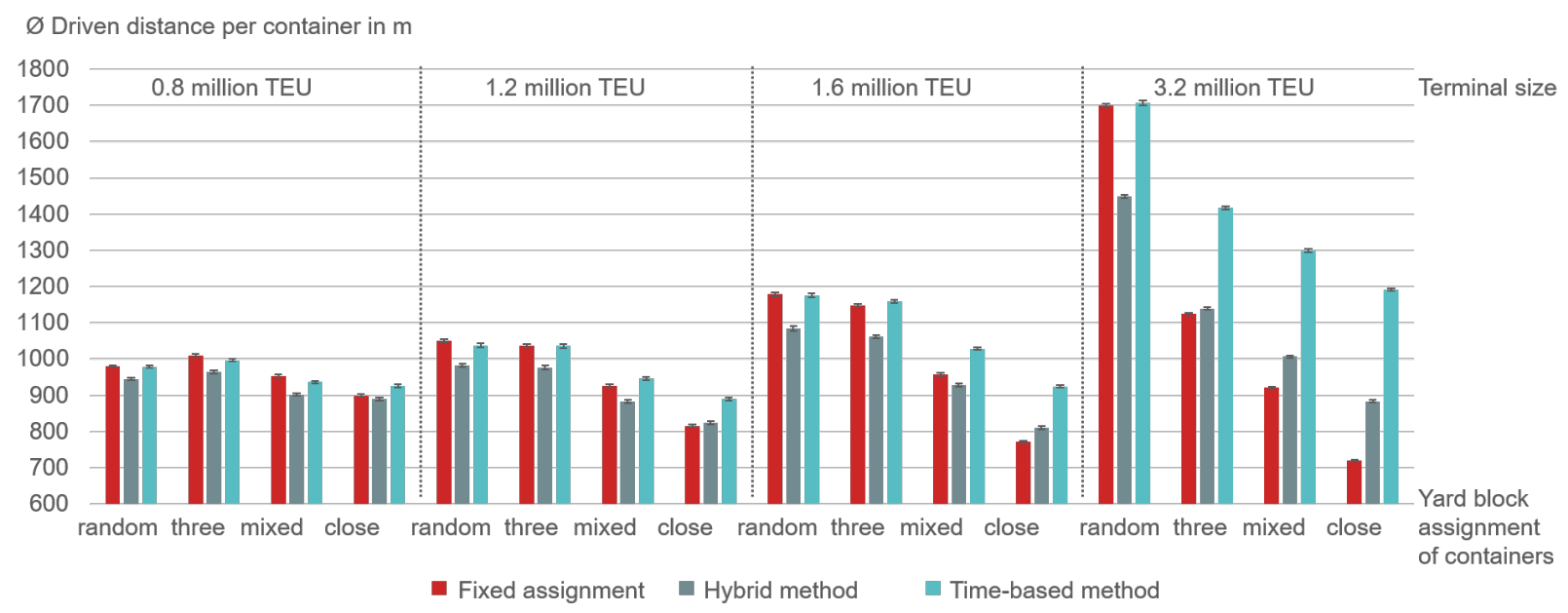

Figure 6: Driven distances depending on terminal size and yard block assignment.

The fluctuations between yard block assignment strategies become greater as the terminal size increases. For the smallest terminal, the range of results averages $9 \%$. For both medium-sized terminals the average range increases to $19 \%$ and $31 \%$, respectively. For the largest terminal, the average range is $58 \%$. In all cases, the range for the fixed assignment is greater than that of the time-based and the hybrid method. Thus, more flexible dispatching methods seem to be better able to compensate for an unfavorable yard block assignment. When the fixed assignment for horizontal transport is used, the correct choice of yard block assignment is particularly important to reduce the distances traveled. In the case of random yard block assignment, the hybrid method leads to the shortest distances traveled in horizontal transport. The advantage increases with the terminal size. For the smallest terminal, the distance saving is $4 \%$ and for both medium-sized terminals it is 7-8\%. For the largest terminal, even up to $15 \%$ of the distances can be reduced when the hybrid dispatching method is chosen. This is plausible, as a larger terminal shows more potential to reduce distances and the hybrid method considers the driven distances as well.

The fixed assignment in horizontal transport together with the close yard block assignment leads to the shortest routes for all terminal sizes. For the two smaller terminals, the hybrid method is close to the fixed assignment, and for the two larger terminals, the distance between the fixed assignment and the hybrid method increases. In case of the fixed assignment and the close yard block assignment, the TT always move back and forth between the STS and the closest yard blocks. The hybrid method minimizes the distances, but if necessary, an order with a greater distance is selected if no order can be chosen for a closer yard block. This has the consequence that with the hybrid method the distances increase, but 
the STS productivity does not decrease. With the fixed assignment, the TT would not carry out the more distant job, but wait for the next job in the vicinity. The more distant job would have to wait longer to be carried out, which leads to a greater risk of waiting times for the STS.

The yard block assignment three leads to shorter distances at the largest terminal compared to random than at the three smaller terminals. There, the results for three and random are closer together. This difference at the largest terminal is probably caused by the fact that the three yard blocks, each assigned to an STS, are located slightly closer to the respective STS than the average of the random yard block assignment. This underlines the relevance of a favorable storage block assignment.

\section{CONCLUSION}

Horizontal transport is the link between sea-side handling of container ships and the storage area. Delays in horizontal transport can result in a decelerated container handling at the quay. Efficient dispatching in horizontal transport is, therefore, an important element in the successful management of container terminals. To ensure efficient horizontal transport, the relationships between dispatching methods for horizontal transport orders, terminal parameters, and the performance of container terminals have been investigated. The yard block assignment and the dispatching method in horizontal transport can be changed comparatively easily, so that a good combination can be found depending on the prioritized terminal objectives (Figure 7).
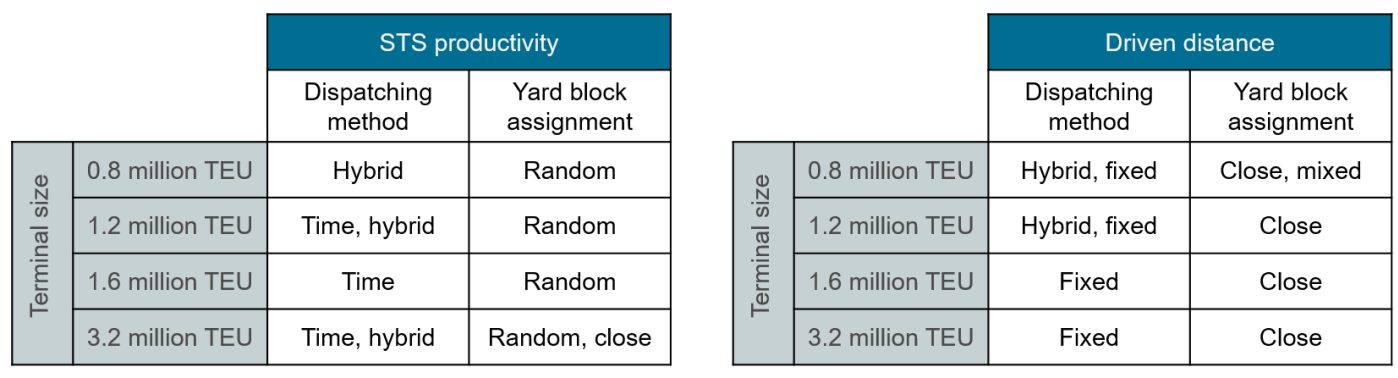

Figure 7: Best dispatching method and yard block assignment depending on the terminal size.

For example, a terminal can focus primarily on STS productivity when a ship needs to be unloaded and loaded quickly. In this case, the time-based and the hybrid dispatching method are good choices in combination with a random yard block assignment. Especially for large terminals also the close yard block assignment helps to reduce waiting times for the STS. If handling is less time-critical, it can be reasonable to save energy and, thus, costs over the distances traveled. This can be achieved by the fixed and - for smaller terminals - also by the hybrid method in combination with a close yard block assignment. For the smallest terminal, also the mixed yard block assignment leads to short distances.

\section{REFERENCES}

Bian, Z., Y. Yang, W. Mi, and C. Mi. 2015. "Dispatching Electric AGVs in Automated Container Terminals with Long Travelling Distance". Journal of Coastal Research 73:75-81.

Brinkmann, B. 2011. "Operations Systems of Container Terminals: A Compendious Overview". In Handbook of Terminal Planning, edited by J. W. Böse, Operations Research/Computer Science Interfaces Series, 25-39. New York: Springer.

Briskorn, D., A. Drexl, and S. Hartmann. 2006. "Inventory-based Dispatching of Automated Guided Vehicles on Container Terminals". OR Spectrum 28(4):611-630.

Carlo, H. J., I. F. Vis, and K. J. Roodbergen. 2014. "Transport Operations in Container Terminals: Literature Overview, Trends, Research Directions and Classification Scheme”. European Journal of Operational Research 236(1):1-13.

Choe, R., H. Cho, T. Park, and K. R. Ryu. 2012. "Queue-based Local Scheduling and Global Coordination for Real-time Operation Control in a Container Terminal”. Journal of Intelligent Manufacturing 23(6):2179-2192. 
Schwientek, Lange, and Jahn

Corman, F., J. Xin, R. R. Negenborn, A. D’Ariano, M. Samà, A. Toli, and G. Lodewijks. 2016. "Optimal Scheduling and Routing of Free-range AGVs at Large Scale Automated Container Terminals". Periodica Polytechnica Transportation Engineering 44(3):145-154.

Dragovic, B., E. Tzannatos, and N. K. Park. 2017. "Simulation Modelling in Ports and Container Terminals: Literature Overview and Analysis by Research Field, Application Area and Tool". Flexible Services and Manufacturing Journal 29(1):4-34.

Dulebenets, M. 2016. "A New Simulation Model for a Comprehensive Evaluation of Yard Truck Deployment Strategies at Marine Container Terminals". Open Science Journal 1(3):1-28.

Gharehgozli, A. H., D. Roy, and R. B. de Koster. 2016. "Sea Container Terminals: New Technologies and OR Models". Maritime Economics \& Logistics 18(2):103-140.

Grunow, M., H.-O. Günther, and M. Lehmann. 2006. "Strategies for Dispatching AGVs at Automated Seaport Container Terminals". OR Spectrum 28(4):587-610.

He, J., Y. Huang, W. Yan, and S. Wang. 2015. "Integrated Internal Truck, Yard Crane and Quay Crane Scheduling in a Container Terminal Considering Energy Consumption”. Expert Systems with Applications 42(5):2464-2487.

Klerides, E., and E. Hadjiconstantinou. 2011. "Modelling and Solution Approaches to the Multi-load AGV Dispatching Problem in Container Terminals". Maritime Economics \& Logistics 13(4):371-386.

Liu, C.-I., and P. Ioannou. 2002. "A Comparison of Different AGV Dispatching Rules in an Automated Container Terminal". In IEEE 5th International Conference on Intelligent Transportation Systems. September $3^{\text {rd }}-6^{\text {th }}$, Singapore, 880-885.

Schwientek, A. K., F. Branding, A.-K. Lange, and C. Jahn. 2018. "Zuordnungsmethoden bei Dual-Load Fahrzeugen im Horizontalverkehr von Container Terminals". In Tagungsband ASIM 2018 - 24. Symposium Simulationstechnik. October $4^{\text {th }}-5^{\text {th }}$, Hamburg, Germany, 157-166.

Schwientek, A. K., A.-K. Lange, and C. Jahn. 2017. "Literature Classification on Dispatching of Container Terminal Vehicles". In Proceedings of the Hamburg International Conference of Logistics HICL. October $12^{\text {th }}-13^{\text {th }}$, Hamburg, Germany, 3-36.

Schwientek, A. K., A.-K. Lange, and C. Jahn. 2018. "Simulation-Based Analysis of Dispatching Methods on Seaport Container Terminals". In Proceedings of the $6^{\text {th }}$ International Conference on Logistics LDIC. February $20^{\text {th }}-22^{\text {nd }}$, Bremen, Germany, 167-171.

Stahlbock, R., and S. Voß. 2008. "Vehicle Routing Problems and Container Terminal Operations - An Update of Research". In The Vehicle Routing Problem: Latest Advances and New Challenges, edited by B. L. Golden, S. Raghavan, and E. Wasil, 551-589. Boston, MA: Springer US.

Tao, J., and Y. Qiu. 2015. "A Simulation Optimization Method for Vehicles Dispatching among Multiple Container Terminals". Expert Systems with Applications 42(7):3742-3750.

Vis, I. F., and R. B. de Koster. 2003. "Transshipment of Containers at a Container Terminal: An Overview”. European Journal of Operational Research 147:1-16.

Wiese, J., N. Kliewer, and L. Suhl. 2009. A Survey of Container Terminal Characteristics and Equipment Types. Working paper 0901, University of Paderborn, Decision Support \& Operations Research Lab.

Zeng, Q., Z. Yang, and L. Lai. 2009. "Models and Algorithms for Multi-crane Oriented Scheduling Method in Container Terminals". Transport Policy 16(5):271-278.

\section{AUTHOR BIOGRAPHIES}

ANNE KATHRINA SCHWIENTEK is a scientist at the Institute of Maritime Logistics at the Hamburg University of Technology. She holds a diploma in Industrial Engineering from Karlsruhe Institute of Technology. Her doctoral thesis focuses on the dispatching efficiency of horizontal transport on container terminals. Her research interest is in modeling and simulation, especially in the application areas seaport and inland container terminals with a focus on dispatching strategies and energy management. Her e-mail address is a.schwientek@tuhh.de.

ANN-KATHRIN LANGE is a senior scientist at the Institute of Maritime Logistics at the Hamburg University of Technology. She holds a M.Sc. in Industrial Engineering and Management from the Technical University of Clausthal. Her doctoral thesis deals with the influence of truck appointment systems at seaport container terminals on the parties involved in port-internal container transport. Her research interests lie in particular in the use of simulation and optimization methods to solve problems in the maritime supply chain. Her e-mail address is ann-kathrin.lange@tuhh.de.

CARLOS JAHN is head of the Institute of Maritime Logistics at the Hamburg University of Technology (Germany) and director of the Fraunhofer Center for Maritime Logistics and Services. He earned his doctorate as a research assistant at the Fraunhofer Institute for Factory Operation and Automation (IFF) at the Otto-von-Guericke University in Magdeburg. His research focuses on new concepts, methods, and solutions in the field of maritime logistics, e.g., logistics planning and simulation, logistics process design, and and the analysis of mobility and company data using machine learning. His email address is carlos.jahn@tuhh.de. 